\title{
The Idea of the Selection of PZT-Beam Interaction Forces in Active Vibration Protection Problem
}

\author{
A. BRAŃSKI ${ }^{a, *}$, M. BORKOWSKI ${ }^{a}$ AND S. SzELA ${ }^{b}$ \\ ${ }^{a}$ Laboratory of Acoustics, WEiI Rzeszów University of Technology, W. Pola 2, 35-959 Rzeszów, Poland \\ ${ }^{b}$ Institute of Technology University of Rzeszów, Rejtana 16c, 35-959 Rzeszów, Poland
}

\begin{abstract}
The paper concerns an active vibration protection ( $p$-reduction) of the structure. This problem corresponds to the active vibration reduction ( $a$-reduction). The quantity and effectivness of the $a-/ p$ - reduction, measured with reduction and effectiveness coefficient respectively, depends on, inter alia, the PZT distribution on the structure subdomains with the largest curvatures (quasi optimal distribution) are considered. The aim of this paper is to determine such interacting forces PZT-structure, assuming QO distribution of PZTs, which maximize the effectiveness of $p$-reduction. The beam clamped at one end, vibrating separately with first three modes, is chosen as the research object. The interacting forces are searched requiring that the shear force and bending moment at the clamped side are equal to zero. The total $p$-reduction is achieved for separate modes. Assuming the QO distribution of the PZTs, the best $p$-effectiveness is achieved. The validation of theoretical considerations is confirmed numerically.
\end{abstract}

PACS numbers: 46.70.De, 02.60.Pn, 46.40.-f, 07.10.Fq

\section{Introduction}

One of the essential technique problem is the vibration reduction of the structure. It can be realized with either active vibration reduction ( $a$-reduction) or active vibration protection ( $p$-reduction). Both methods are different, though they have common features. In both methods the vibration reduction is realized by applying PZTs (piezoelectric elements, actuators). What differs the methods is the way of the aim realization. Namely, in $a$-reduction the actuators are initiated after the vibration is settled, whereas in $p$-reduction the actuators are initiated simultaneously with the excitation of the vibration.

The $a$-reduction problem is well known and it is the subject of a lot of papers e.g. [1-4] and the references cited therein. The $p$-reduction problem is less known and it is the subject of this paper.

The quantity of the vibration reduction ( $a$-reduction or $p$-reduction) depends on many factors. The first one is the PZTs geometric-technical features; for materially monolithic PZTs they are described in monographs [1-3]. Lately, piezo-fiber composites (PFCs) take on more and more meaning; their features are described in [5-7].

The second essential factor is appropriate PZT distribution on the structure. It was proved in $[8,9]$, that for $a$-reduction problem, the most effective PZT distribution is in the structure subdomains with the largest curvatures. Such distribution was called quasi-optimal

* corresponding author; e-mail: abranski@prz.rzeszow.pl
(QO) one. In this paper, it is the QO distribution that is used in $p$-reduction problem.

An effectiveness measure of the vibration reduction is an effectiveness coefficient of the vibration reduction (hereafter: effectiveness coefficient) [9]. It is defined as a quotient of reduction coefficient of the vibration reduction (hereafter: reduction coefficient) and the energy to excite the PZTs. It is obvious, that the reduction effectiveness increases if the effectiveness coefficient increases and vice versa. The quantity of the reduction is measured as follows: $(1$ - directly, by the coefficient of the amplitude (value) vibration reduction), (2 - indirectly, by the coefficient of the shear force (value) and bending moment reduction in the clamped side).

The goal of this paper is to determine such forces interacting between PZTs and the structure, assuming QO distribution of PZTs, which maximize the effectiveness of p-reduction.

In the above-mentioned own papers, a right-angled triangle with clamped-free-free (C-F-F) boundary conditions is the research object. It is extraordinary difficult to realize the aim of this paper for the triangular plate. It is considerably easier to do it for the beam. So, the beam clamped at one end is chosen as the research object. It is assumed that the beam is excited with evenly spread and harmonic force. The force acts with first three natural frequencies. The inner damping coefficient of the material is considered. Rectangular, made from monolithic material PZTs are chosen as the actuators.

The energy provided to the system is in the form of voltage applied to the surface of the PZTs. Assuming that the charge is homogenously distributed on the sur- 
faces, as a result of piezoelectric effect, the PZTs interact with the beam with moments of a couple of forces homogenously distributed along the PZTs' edges. Next, these moments are replaced with the couple of forces. The aim of this paper leads to determine just these forces. It is also assumed, that all PZTs are identical from the geometric-technical aspect. From a control point of view, it is very advantageous because the PZTs are excited with the same signal. As a result, the PZTs interact with the beam with the same forces. Values of these forces are derived theoretically, assuming the bending moment and the shear force in the clamped edge are equal to zero. It means that the quantity of the reduction is measured indirectly. The validity of theoretical considerations is confirmed numerically.

To the authors' knowledge, the active vibration protection problem with the appropriate selection of the forces interacting between actuator and beam, assuming QO distribution of the actuators, have not been considered up to now.

\section{Forced beam vibration with damping}

Let the beam be clamped at one side, Fig. 1; geometrical data of the beam: $\ell$ - length, $S=a h-$ surface of the rectangular cross-section, $a$ - width, $h$ - thickness. Moreover, $f=f(x, t)$ - excited distributed force.

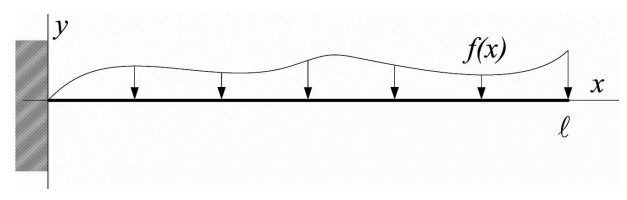

Fig. 1. The geometry of the problem.

The beam vibration equation is given by $[3,10,11]$

$$
E J\left(D_{x}^{4} u+\mu D_{x}^{4}\left(D_{t} u\right)\right)+\rho_{b} S D_{t}^{2} u=-f,
$$

where $u=u(x, t)$ - beam deflection at point $x, u \| y$, $E$ - Young's modulus, $J$ - surface moment of inertia of the beam cross-section: $J=\left(a h^{3}\right) / 12, \rho_{b}$ - mass density, $\mu$ - inner damping factor, $D_{x}^{4}(\ldots)=\partial^{4}(\ldots) / \partial x^{4}$, $D_{t}(\ldots)=\partial(\ldots) / \partial t$.

The boundary conditions are described by the following equations

$$
\begin{aligned}
& u(0, t)=0, \quad D_{x} u(x=0, t)=0, \\
& D_{x}^{2} u(x=\ell, t)=0, \quad D_{x}^{3} u(x=\ell, t)=0 .
\end{aligned}
$$

Beam deflection and rotation angle at the left end of the beam are described respectively by boundary condition (2) $(x=0)$, whereas (3) expresses bending moment and the shear force at the right end $(x=\ell)$. Besides, initial conditions are assumed to be equal to zero.

$$
\begin{aligned}
& u(x, t=0)=0, \\
& D_{t} u(x, t=0)=0 .
\end{aligned}
$$

Let the lateral load force be

$$
f(x, t)=f(x) \mathrm{e}^{\mathrm{i} \omega_{f} t},
$$

where $i=(-1)^{1 / 2}, \omega_{f}$ - excited frequency.

Applying eigenfunction expansion method, the solution of the Eq. (1) is assumed in the following form (it is assumed that after some time, the beam vibrates with excited frequency):

$$
u(x, t)=X(x) \mathrm{e}^{\mathrm{i} \omega_{f} t},
$$

where:

$$
X=\sum_{\nu} C_{\nu} X_{\nu}=\sum_{\nu} X_{f \nu}, \quad \nu=1,2, \ldots, n,
$$

where $C_{\nu}$ - any constants, $X_{\nu}(x)$ - modes (eigenfunctions),

$$
\begin{gathered}
X_{\nu}(x)=c\left(\lambda_{\nu} \ell\right)\left(\sinh \left(\lambda_{\nu} x\right)-\sin \left(\lambda_{\nu} x\right)\right) \\
-s\left(\lambda_{\nu} \ell\right)\left(\cosh \left(\lambda_{\nu} x\right)-\cos \left(\lambda_{\nu} x\right)\right) \\
c\left(\lambda_{\nu} \ell\right)=\cos \left(\lambda_{\nu} \ell\right)+\cosh \left(\lambda_{\nu} \ell\right) \\
s\left(\lambda_{\nu} \ell\right)=\sin \left(\lambda_{\nu} \ell\right)+\sinh \left(\lambda_{\nu} \ell\right)
\end{gathered}
$$

$\left\{\lambda_{\nu}\right\}$ - the set of eigenvalues: $\left\{\lambda_{\nu} \ell\right\}=$ $\{1.8751,4.6941, \ldots,(2 \nu-1) \pi / 2\}$.

The first three modes (9), are depicted in Fig. 2.

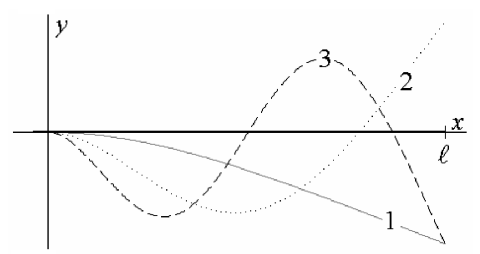

Fig. 2. Normalized modes: $1-X_{1}, 2-X_{2}, 3-X_{3}$.

The constants $C_{\nu}$ are expressed by [10]

$$
C_{\nu}=\frac{1}{\left(1+\mathrm{i} \mu \omega_{f}\right) \omega_{\nu}^{2}-\omega_{f}^{2}} D_{\nu},
$$

where:

$$
\begin{aligned}
& \omega_{\nu}^{2}=\frac{E J}{\rho_{b} S} \lambda_{\nu}^{4}, \\
& D_{\nu}=\frac{1}{\rho_{b} S} \frac{1}{\beta_{\nu}^{2}} I_{\mathrm{D}}, \\
& I_{\mathrm{D}}=-\int_{0}^{\ell} f X_{\nu} \mathrm{d} x, \\
& \beta_{\nu}^{2}=\int_{0}^{\ell} X_{\nu}^{2}(x) \mathrm{d} x .
\end{aligned}
$$

Thus, the problem of the beam vibration with damping, excited with the force $\mathrm{f}(\mathrm{x}, \mathrm{t})$ is solved.

Henceforth, the spread load force with constant amplitude $f_{0}$ is considered, i.e.

$$
f=f(x)=f_{0} \text {. }
$$

Because of the force form (17), and the form of $X_{\nu}(x)(9)$, integral (15) can be calculated analytically.

The solution of the above-mentioned problem is forced vibrations with damping. 


\section{The beam curvature}

As already mentioned in the introduction, the maximal $a$-effectiveness of the triangular plate is obtained, if the PZTs are QO distributed, i.e. they are attached at surface places with maximum curvatures. This idea is applied, to realize the aim of the paper. Hereunder, the curvatures of separate modes of the beam are determined.

Let the beam be checked of pure bending. As an effect of the force acting, the bending beam axis undergoes deformation. The curvature radius of the middle beam line is given by:

$$
\frac{1}{\rho}=-\frac{M}{E J}
$$

where $M_{g}$ — the bending moment. tion:

The curvature of the plane curve is described by equa-

$$
\frac{1}{\rho}= \pm \frac{D_{x}^{2} u}{\left(1+\left(D_{x} u\right)^{2}\right)^{3}}
$$

For small vibrations the slope angle of the tangent to the bending beam axis is small, hence $D_{x} u \ll 1$, and Eq. (19) can be written as:

$$
1 / \rho= \pm D_{x}^{2} u \text {. }
$$

The beam curvature is defined as

$$
\kappa=1 / \rho= \pm D_{x}^{2} u \text {. }
$$

The sign of the curvature is a contractual quantity. If the beam bends downward, then the sign of the curvature is minus and vice versa. Substituting (18) into (21) one gets

$$
\kappa=C_{\nu} D_{x}^{2} X_{\nu}=C_{\nu} \kappa_{\nu} .
$$

For further considerations, it is significant to express the bending moment and the shear force as a function of curvature $[3,10]$

$$
\begin{aligned}
& Q=-E J D_{x}^{3} u=-E J D_{x} \kappa . \\
& M=-E J D_{x}^{2} u=-E J \kappa .
\end{aligned}
$$

In the steady state, the curvature of each mode $X_{\nu}(x)$ is given by

$$
\kappa_{\nu}=D_{x}^{2} X_{\nu} .
$$

\section{Beam vibration reduction via PZT}

As it is already known $[1,2]$, the PZTs interact with the beam with moments of a couple of forces, Fig. 3a. Since the beam vibration equation is the forces equation, then to consider the action of PZTs on the beam, two moments of couple of forces are replaced with two couples of forces, Fig. 3b. Next, the separate forces are considered in the Eq. (1). Hence, the total load of the beam described by Eq. (6) is the sum of the load force expressed by Eq. (17) and the forces interacting between PZTs and the beam, and it is given by

$$
\begin{aligned}
& f(x)=-f_{0} \\
& \quad+\left(f_{\mathrm{P}} \delta\left(x_{\mathrm{P}}-l_{\mathrm{P}}\right)-2 f_{\mathrm{P}} \delta\left(x_{\mathrm{P}}\right)+f_{\mathrm{P}} \delta\left(x_{\mathrm{P}}+l_{\mathrm{P}}\right)\right)
\end{aligned}
$$

An expression in brackets is the sum of interacting forces PZTs-beam, Fig. 3b, $\delta($.$) - Dirac function, a unit$ of all sum elements in Eq. (26) is $[\mathrm{N} / \mathrm{m}]$; hence, the units of $f(x), f_{0}$ and $f_{\mathrm{P}}$ arise [12].

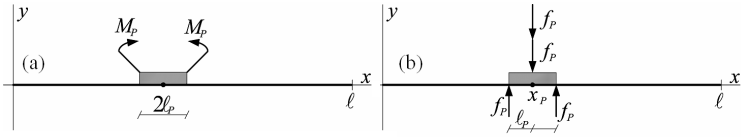

Fig. 3. The idea of the interaction between PZT and the beam.

The integral $I_{\mathrm{D}}$, Eq. (15), for $f(x)$ expressed by Eq. (26), is given by

$$
\begin{aligned}
I_{\mathrm{D}} & =-\int_{0}^{\ell} f X_{\nu} \mathrm{d} x=-f_{0} \int_{0}^{\ell} X_{\nu} \mathrm{d} x \\
& +f_{\mathrm{P}} \int_{0}^{\ell}\left[\delta\left(x_{\mathrm{P}}-l_{\mathrm{P}}\right)-2 \delta\left(x_{\mathrm{P}}\right)\right. \\
& \left.+\delta\left(x_{\mathrm{P}}+l_{\mathrm{P}}\right)\right] X_{\nu} \mathrm{d} x=-f_{0} \int_{0}^{\ell} X_{\nu} \mathrm{d} x \\
& +f_{\mathrm{P}}\left[X_{\nu}\left(x_{\mathrm{P}}-l_{\mathrm{P}}\right)-2 X_{\nu}\left(x_{\mathrm{P}}\right)+X_{\nu}\left(x_{\mathrm{P}}+l_{\mathrm{P}}\right)\right] .
\end{aligned}
$$

The expression in square bracket in Eq. (27) constitutes the second-order central finite difference. Since the distance between nodes $l_{\mathrm{P}}$ is constant, then the difference can be transformed into

$$
\begin{aligned}
& \frac{1}{l_{\mathrm{P}}^{2}}\left[X_{\nu}\left(x_{\mathrm{P}}-l_{\mathrm{P}}\right)-2 X_{\nu}\left(x_{\mathrm{P}}\right)+X_{\nu}\left(x_{\mathrm{P}}+l_{\mathrm{P}}\right)\right] \\
& \quad=D_{x}^{2} X_{\nu}\left(x_{\mathrm{P}}\right),
\end{aligned}
$$

where, according to Eq. (25)

$$
D_{x}^{2} X_{\nu}\left(x_{\mathrm{P}}\right)=\kappa_{\nu}\left(x=x_{\mathrm{P}}\right)=\kappa_{\mathrm{P}} \text {. }
$$

The $\kappa_{\mathrm{P}}$ is the curvature of the mode at the point. The curvatures of the first three modes are depicted in Figs. 4a, 5a, 6a respectively. Substituting Eqs. (28) and (29) into Eq. (27), one obtains

$$
I_{\mathrm{D}}=-f_{0} \int_{0}^{\ell} X_{\nu} d x+f_{\mathrm{P}} l_{\mathrm{P}}^{2} \kappa_{\mathrm{P}}=I_{\mathrm{D} 0}+I_{\mathrm{DP}} .
$$

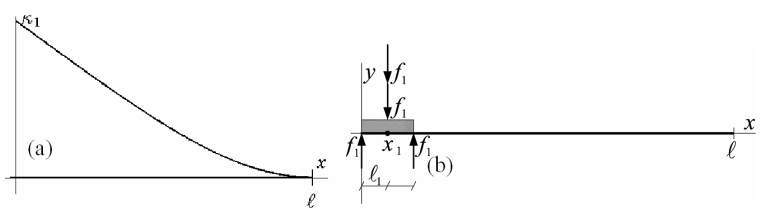

Fig. 4. Curvature $\kappa_{1}(x)$ (a); and QO PZT distribution for the 1st mode (b).

The sign of $I_{\mathrm{DP}}$ depends on:

- the direction of the forces $f_{\mathrm{P}}$ on the PZT edges assuming, that $\kappa_{\mathrm{P}}$ is always positive, or

- the sign of $\kappa_{\mathrm{P}}$ assuming, that $f_{\mathrm{P}}$ is always positive.

At first, the former rule, which is more natural, is assumed: now $2 f_{\mathrm{P}}$ is placed at the point of local extreme of the mode with the opposite direction to the beam deflection $u(x, t)$, Fig. 3b. In turn, the forces $f_{\mathrm{P}}$ on the PZT 


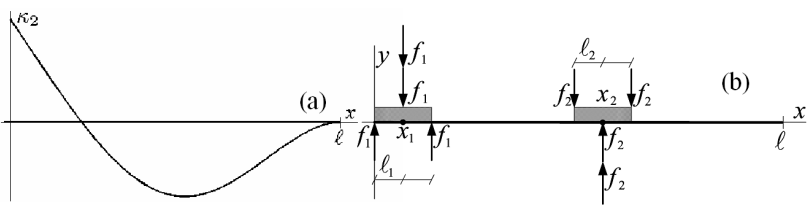

Fig. 5. Curvature $\kappa_{2}(x)$ (a); and QO PZT distribution for the 2nd mode (b).

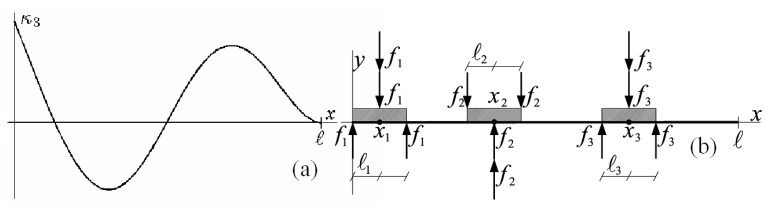

Fig. 6. Curvature $\kappa_{3}(x)(\mathrm{a})$; and QO PZT distribution for the 3rd mode (b).

edges are in opposite direction to $2 f_{\mathrm{P}}$; it is the direction of the $f_{\mathrm{P}}$ on the PZT edges that decides on the sign of $I_{\mathrm{DP}}$. In other words, $I_{\mathrm{DP}}$ is positive, if the vectors $f_{\mathrm{P}}$ and the beam deflection $u(x, t)$ are in the same direction.

Equation (30) can be generalized to the case, when vibration reduction is carried out with many PZTs. In this case

$$
\begin{aligned}
I_{\mathrm{D}} & =I_{\mathrm{D} 0}+f_{1} \ell_{1}^{2} \kappa_{1}-f_{2} \ell_{2}^{2} \kappa_{2}+\ldots \\
& =I_{\mathrm{D} 0}+\sum_{\mathrm{P}}(-1)^{\mathrm{P}+1} f_{\mathrm{P}} \ell_{\mathrm{P}}^{2} \kappa_{\mathrm{P}}=I_{\mathrm{D} 0}+I_{\mathrm{DP}}, \\
& P=1,2, \ldots, n_{\mathrm{P}}
\end{aligned}
$$

where, $n_{\mathrm{P}}$ - number of PZTs or the number of the local curvature extremes of separate modes. It is obvious, that the signs $I_{\mathrm{DP}}$ are positive and negative alternately; in Eq. (31), it is assumed that $I_{\mathrm{D} 1}$ is positive.

If all PZTs have the same size $l_{\mathrm{P}}$ and they act on the beam with the same forces $f_{\mathrm{P}}$, then instead of Eq. (31) one can apply

$$
\begin{gathered}
I_{\mathrm{D}}=I_{\mathrm{D} 0}+f_{\mathrm{P}} \ell_{\mathrm{P}}^{2} \sum_{\mathrm{P}}(-1)^{\mathrm{P}+1} \kappa_{\mathrm{P}}, \\
P=1,2, \ldots, n_{\mathrm{P}}
\end{gathered}
$$

As it can be seen, the second rule of determining the sign of $I_{\mathrm{DP}}$ in Eq. (32) has appeared; the sign of the curvature $\kappa_{\mathrm{P}}$ decides about the sign of particular components of $I_{\mathrm{DP}}$.

\section{Selection of forces amplitudes of the PZT-beam interaction}

The $f_{\mathrm{P}}$ force amplitudes are selected for $p$-reduction problem. The direct aim of the vibration reduction is minimization of the vibration amplitude, and the indirect goal is to minimize the shear force and the bending moment at beam cross-sections especially at this one, which decide about the strength of the structure - here, in the clamped side. The best situation is when these quantities are minimized to zero. For selected modes, the shear force and the bending moment are given by Eqs. (23) and (24) respectively.

An idea of the problem solution is as follows. Bending moment $M(x)$ equals to zero, when $\kappa(x)=0$. Whereas the shear force $Q(x)$ equals to zero, when the curvature $\kappa(x)$ is constant or takes its extreme. Both quantities equal to zero when $\kappa(x) \equiv 0$. But from Eq. (21) appears, that because of the shape $X_{\nu}(x)$, the $\kappa(x)$ may not be equal to zero at all points of the beam. So, only the condition $C_{\nu}=0$ ensures $M(x)=0, Q(x)=0$ simultaneously.

From Eq. (12) it appears, that $C_{\nu}=0$ when $D_{\nu}=0$, Eq. (14). Hence, omitting the constant $1 /\left(\rho S \beta_{\nu}^{2}\right)$, on the basis of Eq. (15), the following condition of zeroing both $M(x)$ and is obtained

$$
I_{\mathrm{D}}=-\int_{0}^{\ell} f X_{\nu} \mathrm{d} x=0 .
$$

For the case under consideration, $I_{\mathrm{D}}$ is given by Eq. (32). From (33), via (32), one can obtain

$$
f_{\mathrm{P}}=-\frac{I_{\mathrm{D} 0}}{\ell_{\mathrm{P}}^{2} \sum_{\mathrm{P}}(-1)^{\mathrm{P}+1} \kappa_{\mathrm{P}}} \text {. }
$$

Equation (34) describes the value of the forces with which $n_{\mathrm{P}}$ elements act on the beam vibrating with separate mode $X_{\nu}(x)$. In practice it means, that under these forces $f_{\mathrm{P}}$ the beam does not vibrate at all.

\section{Numerical calculations}

In this section the validity of the theoretical considerations by numerical examples is confirmed. In numerical calculations the following data are assumed: $\ell=0.27 \mathrm{~m}$, $a=0.025 \mathrm{~m}, h=0.001 \mathrm{~m}, E=2.05 \times 10^{11} \mathrm{~Pa}$, $\mu=1.94 \times 10^{-4} \mathrm{~s}, \rho_{b}=7.8 \cdot 10^{3} \mathrm{~kg} / \mathrm{m}^{3}, f_{0}=0.02 \mathrm{~N} / \mathrm{m}$. It is assumed, that sizes of all PZTs are the same, i.e. $\ell_{1}=\ell_{2}=\ell_{3}=0.05 \ell$, and all PZTs are excited by the same signal. This assumption concerns the second and the third mode.

At first, according to Eq. (34), the curvatures of beam deflection line are calculated for the first three modes; the results are depicted in Figs. 4a, 5a, 6a. For each mode, at the QO subdomain only one PZT is bonded, see Figs. 4b, $5 \mathrm{~b}, 6 \mathrm{~b}$. The forces $2 f_{\mathrm{P}}$ are placed at the points of the $\mathrm{QO}$ subdomain: the 1st mode $-\left\{x_{\mathrm{P}}\right\}=x_{1}=\ell_{1}$, the 2nd mode $-\left\{x_{\mathrm{P}}\right\}=\left\{x_{1}, x_{2}\right\}=\left\{\ell_{1}, 0.1429\right\}$, the 3rd mode $-\left\{x_{\mathrm{P}}\right\}=\left\{x_{1}, x_{2}, x_{3}\right\}=\left\{\ell_{1}, 0.0831,0.1914\right\}$. Since the first curvature in all three modes is at the point $x=0$, then the first PZTs has to be moved away from clamped side within necessary distance $\ell_{1}$.

In accordance with Eq. (34) the values of interacting forces between PZTs and the beam are: for the 1st mode - $f_{1}=0.2583$, for the 2 nd mode $-f_{1}=f_{2}=0.0144$, for the 3rd mode $-f_{1}=f_{2}=f_{3}=0.0022$. Next, for the first and the second mode, the values of these forces were determined numerically according to the following procedure. For the set of the values $\left\{f_{\mathrm{P}}\right\}$, on the basis Eqs. (23) and (24), the sets of values of $\{|Q|\}$ and $\{|M|\}$ are calculated; the results are shown in Figs. 7 and 8 
respectively. More readable and evocative is Fig. 7 which concerns the first mode. As it can be seen in this figure, at first, values of $|Q|$ and $|M|$ decrease. For $f_{1}=0.2583$ both these quantities are equal to zero; this is exactly the same value of $f_{1}$ as calculated from Eq. (34). Next, both quantities increase, if the $f_{\mathrm{P}}$ increase.

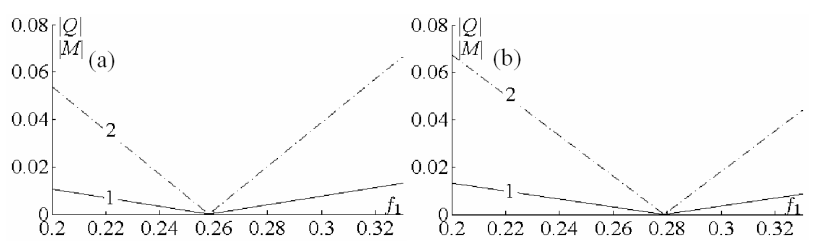

Fig. 7. $1-\left|M\left(f_{1}\right)\right|, 2-\left|Q\left(f_{1}\right)\right|$ for QO PZT distribution (a); $1-\left|M\left(f_{1}\right)\right|, 2-\left|Q\left(f_{1}\right)\right|$ for non-QO PZT distribution (b).

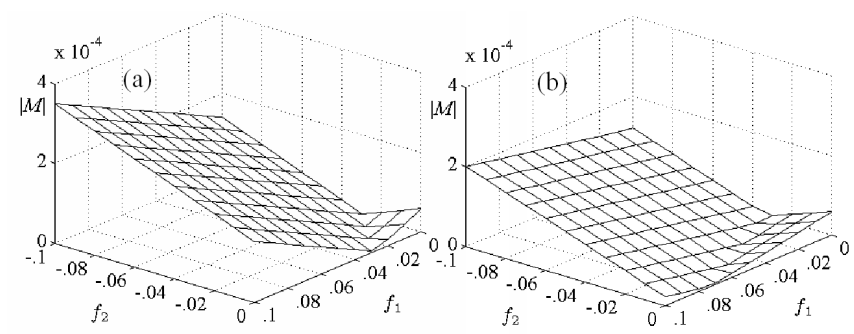

Fig. 8. $\left|M\left(f_{1}, f_{2}\right)\right|\left|Q\left(f_{1}\right)\right|$ for QO PZT distribution (a); $\left|M\left(f_{1}, f_{2}\right)\right|$ for non-QO PZT distribution (b).

For comparison, PZT is placed outside the QO subdomain, e.g. $\left\{x_{\mathrm{P}}\right\}=x_{1}=\ell / 10$. The results are presented in the Fig. 7b. As it can be seen, in this case the total reduction of $|Q|$ and $|M|$ may also be achieved. However, it has to be done with larger force $f_{1}=0.2789$.

The identical numerical simulation is carried out for the second mode. The results for the bending moment are shown in Fig. 8. Figures with surfaces of the shear force $\left|Q\left(f_{1}, f_{2}\right)\right|$ are omitted because they differ from $\left|M\left(f_{1}, f_{2}\right)\right|$ only in different angle of slope (the same situation as in the case of the first mode). As it can be observed, when $f_{1}=f_{2}=0.0144$ both $|Q|=0$ and $|M|=0$; this is the value that is obtained in accordance with Eq. (34). Both these quantities are also equal to zero when the values of $f_{1}$ and $f_{2}$ are different; one can find these values on the line $|Q|=0$ or $|M|=0$ in Fig. 8a. It is also interesting, that $|Q|=0$ and $|M|=0$ can be achieved, even if only one PZT is attached. For this case the value of the force interacting between PZT and the beam may be read from the axis either $f_{1}$ or $f_{2}$ in Fig. 8a $\left(f_{1}=0.0279\right.$ and $\left.f_{2}=0.0295\right)$.

Quite similar like for the first mode, Fig. 7b, fulfilling the condition $|Q|=0$ and $|M|=0$ is achieved, if one or several PZTs is attached outside the QO subdomains; here $\left\{x_{\mathrm{P}}\right\}=\left\{x_{1}, x_{2}\right\}=\{0.0405,0.1699\}$. But at that time the values of $f_{1}$ and $f_{2}$ are much bigger, Fig. $8 \mathrm{~b}$, than the ones obtained for QO distribution, Fig. 8a; they are $f_{\mathrm{P}}=f_{1}=f_{2}=0.0226$

The similar numerical simulations can be done for higher modes, however their graphical presentations are complicated.

What still remains is to determine p-reduction coefficient and p-effectiveness coefficient. The former is defined as follows [9]

$$
R_{\alpha}=\frac{\alpha-\alpha_{\mathrm{R}}}{\alpha},
$$

where $\alpha$ i $\alpha_{\mathrm{R}}$ - values of quantities calculated without and with PZT respectively, $\alpha=\{Q, M\}$. If vibration reduction is complete then $R_{\alpha}=1$.

The latter coefficient is given by the formula [9]

$$
R_{\mathrm{E} ; \alpha}=R_{\alpha} / E_{n},
$$

where $E_{n}$ - an amount of the energy provided to the system in order to excite PZT. Energy provided to the system is translated into couples of forces, subsection 4 . Therefore, the energy $E_{n}$ in Eq. (36) may be replaced with these forces, hence

$$
R_{\mathrm{E} ; \alpha}=R_{\alpha} / f_{\mathrm{PZT}}
$$

where $f_{\mathrm{PZT}}=\sum_{\mathrm{P}} f_{\mathrm{P}}, P=1,2, \ldots n_{\mathrm{P}}, n_{\mathrm{P}}$ - number of PZTs in the system. Hereafter in the denominator in Eq. (37), for each PZT only one value of $f_{\mathrm{P}}$ is taken into account. The results will be comparable, if this rule is retained for each mode. Next, the p-effectiveness coefficient only for bending moment $M$ at the clamped side, and only for the first mode is calculated. The calculations for the shear force $Q$ and higher modes are omitted. This is because an idea of these calculations is analogical; however the graphical presentation of the results is very complicated.

The p-effectiveness coefficient is calculated for three cases:

1. PZT is placed in QO domain,

2. PZT is placed in the middle of the beam,

3. PZT is placed at three quarters of the beam.

For these cases the interacting forces between PZT and beam are calculated, i.e. $\left\{f_{1 ; 1}=f_{1 ; \mathrm{QO}}, f_{1 ; 2}, f_{1 ; 3}\right\}=$ $\{0.2583,0.7084,2.4722\}$ for which total $p$-reduction of the bending moment $M$ is achieved; where the index e.g. 1; QO means first mode and QO-quasi-optimal distribution of the PZTs.

Next, for each case, accordingly to Eq. (35), the $p$ -reduction coefficient was calculated in terms of position of PZT centre, $x_{1} \in\left[\ell_{1}, \ell-\ell_{1}\right]$, the results are shown in Fig. 9a. In accordance with Eq. (37), the $p$ -effectiveness coefficients $R_{\mathrm{E} ; \mathrm{M}}=\left\{R_{\mathrm{E} ; 1}, R_{\mathrm{E} ; 2}, R_{\mathrm{E} ; 3}\right\}=$ $\{3.8717,1.4107,0.4025\}$ are calculated from Eq. (37) at points where $R_{\mathrm{M}} \equiv 1$, the results are presented in Fig. $9 \mathrm{~b}$.

As it can be seen in Fig. 9, the best $p$-effectiveness is obtained when PZT is placed in QO subdomain and $f_{1 ; 1} \equiv f_{1 ; \mathrm{QO}}$. This way, the goal of the paper was achieved. 


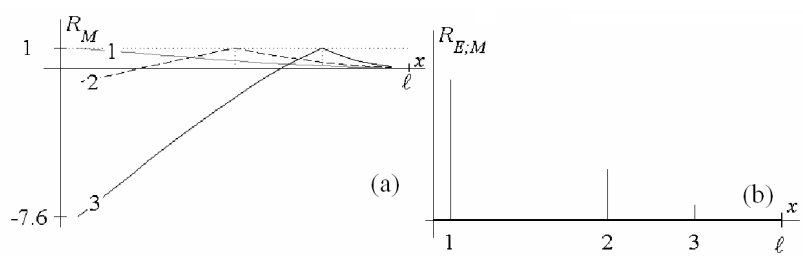

Fig. 9. $\quad R_{\mathrm{M}}(\mathrm{a})$; and $R_{\mathrm{E} ; \mathrm{M}}(\mathrm{b})$ in terms of PZT location; $\{1,2,3\}$ for $\left\{f_{1 ; 1} \equiv f_{1 ; \mathrm{QO}}, f_{1 ; 2}, f_{1 ; 3}\right\}$.

\section{Conclusions}

The conclusions enumerated below arise from theoretical and numerical considerations. It should be emphasized that they are formulated in the following assumption: all PZTs are identical on the grounds of geometric-technical aspects. Consequently, the amplitudes of the interacting forces between PZTs and beam have the same values. Working on this assumption the first conclusion is formulated:

1. The $f_{\mathrm{P}}$ value providing the total p-reduction can be calculated from the condition of zeroing of the shear forces and bending moments at the clamped side. The numerical results computed by Eqs. (28) and (29) are depicted in Figs. 7 and 8.

It is worth to know, that an effect of total vibration $p$-reduction can be also achieved, when two or more different PZTs are used, i.e. for different values of $f_{\mathrm{P}}$. But the analytical calculations of the values $f_{\mathrm{P}}$ were not successful; they are only obtained by numerical simulation, Fig. 8 .

The most favorable case is when the total $p$-reduction is attained providing to the system minimum energy. In other words, the $p$-reduction should be carried out in such a way that the $p$-effectiveness coefficient takes maximum. In presented problem it leads to determining minimal force $f_{\mathrm{P}}$ ensuring total $p$-reduction. The considerations lead to the second conclusion:

2. The best p-effectiveness is achieved, if PZTs are QO distributed.
An analogous conclusion, was drawn for a-reduction for the triangular plate with the $\mathrm{C}-\mathrm{F}-\mathrm{F}$ boundary conditions. But there, the $f_{\mathrm{P}}$ forces were assumed arbitrary for separate modes, i.e. they were not calculated analytically. Furthermore, the total $a$-reduction was not obtained; it seems that it is impossible there.

In this paper the vibration $p$-reduction was performed only for separate modes. The research of the $p$-effectiveness for general beam vibration and also for different, in geometric-technical regards, PZTs, may be very interesting. In these cases, PZT-beam interaction forces would be different. The research on these problems is carried on.

\section{References}

[1] C.R. Fuller, S.J. Elliot, P.A. Nielsen, Active control of vibration, Academic Press, London 1997.

[2] C.H. Hansen, S.D. Snyder, Active control of noise and vibration, E\&FN SPON, London 1997.

[3] M. Pietrzakowski, Active damping of transverse vibration using distributed piezoelectric elements, OWPW, Warszawa 2004.

[4] J. Wiciak, Vibration of structural acoustics control selected aspects, AGH, Kraków 2008.

[5] W. Kurnik, A. Tylikowski, Mechanics of laminate elements, OWPW, 1997 (in Polish).

[6] P.M. Przybyłowicz, Piezoelectric vibration control of rotating control, OWPW, Warszawa 2002.

[7] A. Tylikowski, P.M. Przybyłowicz, Nonclassical piezoelectric materials in stabilization and vibration damping, IPBMPW, 2004 (in Polish).

[8] A. Brański, S. Szela, Archives of Control Sciences, 17, 427 (2007).

[9] A. Brański, S. Szela, Archives of Acoustics, 33, 413 (2008).

[10] S. Kaliski, Vibration and waves (in Polish), PWN, Warszawa 1986.

[11] E. Kącki, Partial differential equations (in Polish), WNT, Warszawa 1992.

[12] M. Kozień, Acoustic radiation of plates and shallow shells (in Polish), PK, Monografia 331, Kraków 2006. 